Thorax, 1979, 34, 690-691

\title{
Unilateral pulmonary oedema associated with old poliomyelitis
}

\author{
V HAJIROUSSOU AND R C JOSHI \\ From the Medical Unit, Manor Hospital, Walsall, West Midlands, UK
}

The characteristic radiological appearance of pulmonary oedema is the "batwing" shadow. Unusual distributions of oedema, however, is occasionally seen. Unilateral pulmonary oedema has been reported in patients with asymmetrical blood flow as in congenital heart disease with aortopulmonary shunt (Albers and Nadas, 1967), unilateral pulmonary emphysema (Saleh et $a l, 1974)$, and after rapid expansion of the lung on aspiration of air or fluid from the pleural cavity.

We report a patient in whom weakness of the left intercostal muscles due to old poliomyelitis was considered responsible for the localisation of oedema fluid in the right lung during episodes of left ventricular failure.

\section{Case report}

A 56-year-old labourer presented on 28 November 1976 with pronounced breathlessness of a few hours' duration. At the age of $2 \frac{1}{2}$ years he had had poliomyelitis affecting the left arm and right leg. On admission his respiratory rate was $40 / \mathrm{min}$, blood pressure $130 / 80 \mathrm{mmHg}$, and pulse $100 / \mathrm{min}$, regular, and of low volume. A few crackles were heard at both lung bases. He had mild kyphoscoliosis and wasting of the muscles of the left arm and right leg. A chest radiograph (figure) showed diffuse shadowing in the right lung consistent with oedema. An electrocardiogram



Chest radiograph soon after admission showing oedema of the right lung. showed left bundle-branch block. He was treated with frusemide and digoxin. Clinical improvement was $x$ noticeable within a few hours. A repeat chest radio- $\underset{A}{\omega}$ graph three days later showed complete resolution of $c$ the oedema. Ten months later he was readmitted with $\delta$ symptoms and signs similar to those of the previous

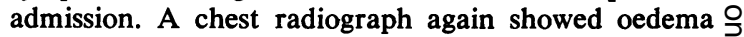
confined to the right lung. The electrocardiogram showed no change but serial tests of aspartate tran- $\bigcirc$ saminase and urea stable lactic dehydrogenase were suggestive of myocardial infarction. He improved with intravenous frusemide, and a chest radiograph taken 24 hours later was clear.

Over the next four months he had three further admissions to hospital with acute breathlessness. On each occasion the chest radiograph showed oedema confined to the right lung. Respiratory function tests showed normal vital capacity and forced expiratory $\bar{\partial}$ volume in one second. A perfusion scan of the lungs

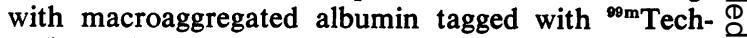
netium showed slightly decreased perfusion of the $\overrightarrow{\vec{F}}$ left lung. Xenon washout scan showed no gross abnormality.

The patient died suddenly at home on 11 October 1977. At necropsy there was severe atheroma of the coronary arteries, biventricular hypertrophy, and wasting of the left intercostal muscles. Histological examination showed pronounced atrophy, fibrosis, and focal calcification of the left intercostal muscles and extensive scarring of the myocardium due to previous infarction. There was no significant abnormality in the vessels or acinar structures of the two lungs.

\section{Discussion}

The mechanism for the development of pulmonary oedema is not yet fully known. The general fluid trans- $\widetilde{N}$ port equation of Starling is thought to apply in the $N$ lungs. Under normal conditions this envisages an $\mathrm{N}$ equilibrium of hydrostatic and osmotic pressures in $\omega$ the intravascular and interstitial spaces. There are $\underset{x}{ }$ several features distinctive to the pulmonary circula- 0 tion, however, which may affect the transport of fluid $\overparen{D}$ across the vascular walls in the lungs. Pulmonary circulation is a low pressure system, and small changes $\square$ in the interstitial space pressure exert a greater effect on the blood flow. The pressure in this space is more negative than in most peripheral tissues and a mean value lower than $-10 \mathrm{mmHg}$ is widely accepted. It has been shown experimentally that this pressure falls with inflation (Meyer et al, 1968), while the blood 
flow in the extra-alveolar vessels increases. The capillaries in the alveolar walls are subjected to alveolar pressure, while the vessels in the extra-alveolar space are exposed to the negative interstitial pressure. Even these larger extra-alveolar vessels have very thin walls and contribute significantly to transport of fluid into the interstitium (Iliff, 1971).

Local differences in the intravascular and interstitial pressures as a result of uneven lung inflation may influence the distribution of pulmonary oedema. Gravity and increased capillary permeability due to endothelial damage may also influence the distribution of oedema fluid.

Our patient had recurrent episodes of left ventricular failure, secondary to coronary artery disease. Oedema confined to the right lung was seen on each occasion. This recurrent presentation makes gravity a less likely cause for the localisation of oedema. Necropsy failed to show a significant difference in the size and structure of pulmonary vessels on either side and the acinar structure of both lungs was normal. There was considerable wasting of the left intercostal muscles, however, which, most probably, had been the result of poliomyelitis in early childhood, together with wasting of muscles in the right leg and left arm. We suggest therefore that relative restriction of inflation of the left lung due to weak left intercostal muscles protected that lung from accumulation of oedema fluid. Restricted inflation may also explain the reduced perfusion in the left lung seen on the perfusion scan. No report of a similar kind could be traced, although Steckel (1973) observed the protective effect of relative deflation of the lung against accumulation of oedema fluid in two patients with left heart failure and pneumothorax.

We gratefully acknowledge the help given by $\mathrm{Dr} I$ Goldsmith, consultant pathologist, and Dr P Carpenter, consultant radiologist.

\section{References}

Albers, W H, and Nadas, A S (1967). Unilateral chronic pulmonary edema and pleural effusion after systemic-pulmonary artery shunt for cyanotic congenital heart disease. American Journal of Cardiology, 19, 861-866.

Iliff, L D (1971). Extra-alveolar vessels and edema development in excised dogs' lungs. Circulation Research, 28, 524-532.

Meyer, B J, Meyer, A, and Guyton, A C (1968). Interstitial fluid pressure-V. Negative pressure in the lungs. Circulation Research, 22, 263-271.

Saleh, M, Miles, A I, and Lasser, R P (1974). Unilateral pulmonary edema in Swyer-James syndrome. Chest, 66, 594-597.

Steckel, R J (1973). Unilateral pulmonary edema after pneumothorax. New England Journal of Medicine, 289, 621-622.

Requests for reprints to: Dr R C Joshi, Manor Hospital, Walsall, West Midlands. 